\title{
Data models for the Compton camera acquisition and their influence on the reconstructed images
}

Voichița Maxim, Xavier Lojacono, Estelle Hilaire (CREATIS) Jean-Luc Ley, Denis Dauvergne, Etienne Testa (IPNL)

\author{
Université de Lyon, France
}

Geneva, February 10-14, 2014 


\section{Summary}

(1) SPECT imaging with the Compton camera

(2) From the events to the image : the system matrix

(3) Image reconstruction : list-mode MLEM algorithm

(4) Numerical results 


\section{Summary}

(1) SPECT imaging with the Compton camera

(2) From the events to the image: the system matrix

(3) Image reconstruction: list-mode MLEM algorithm 


\section{SPECT imaging with the Compton camera}

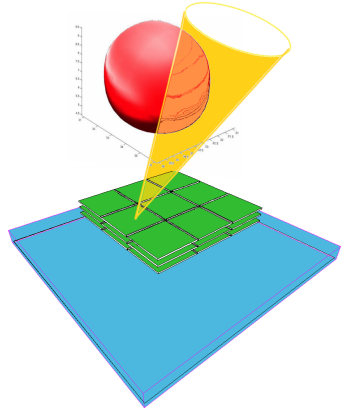

- Source of $\gamma$ particles : emission at some point $V_{0}$ and initial energy $E$

- Scatterer : first interaction (Compton scattering) at some $V_{1}$ and energy transmitted to an electron denoted $E_{1}$

- Absorber: second interaction at some $V_{2}$ (photoelectric absorption) and energy $E_{2}$

- Projection pattern : integral on the surface of a cone

For multiple scatterings, $V_{2}$ is the second $\cos \beta=1-\frac{m_{e} c^{2} E_{1}}{\left(E-E_{1}\right) E}$

(Compton scattering angle) Compton interaction and $E_{2}$ estimates $E-E_{1}$.

Event $\mathscr{E}=\left(V_{1}, V_{2}, E_{1}, E_{2}\right)$ associated to a Compton cone $\mathscr{C}\left(V_{1}, V_{2}, \beta\right)$ 


\section{Applications}

The sensitivity of the Compton camera is superior to the one of the Anger camera by 1-2 orders of magnitude.

- Imaging of poly-energetic sources

- Imaging of sources with energies $\sim 1 \mathrm{MeV}$

- 3D imaging with a single camera

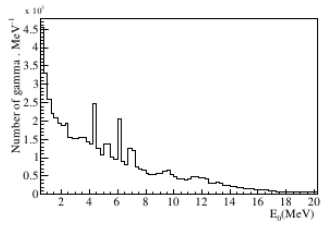

Emission spectrum of prompt- $\gamma$ particles between 0.5 and $20 \mathrm{MeV}$ obtained with a carbon ion beam at $350 \mathrm{MeV} / \mathrm{u}$ irradiating a water phantom obtained with
with $10^{5}$ ions.
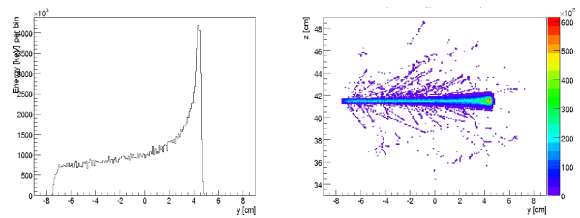

PMMA-sphere irradiated by a proton beam (140 MeV), Edep.

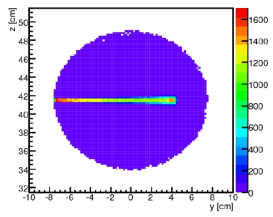

Last interaction of $\gamma$ particles that escape the phantom.

("A tracking Compton-scattering imaging system for hadron therapy monitoring", M. Frandes,

A. Zoglauer, V. Maxim, R. Prost, IEEE TNS, 2010) 


\section{Summary}

(1) SPECT imaging with the Compton camera

(2) From the events to the image: the system matrix

(3) Image reconstruction: list-mode MLEM algorithm 
Challenge: from the events to the image

Requires a model for the conditional probability of $e=\left(V_{1}, V_{2}, E_{1}, E_{2}\right)$ given the emission point $V_{0}$,

$$
p\left(\mathscr{E}=e \mid V_{0}\right) .
$$

We choose to focus on the geometrical parameters and to ignore the influence of scattering/absorption probabilities. When real positions of interaction and real energies are supposed to be measured,

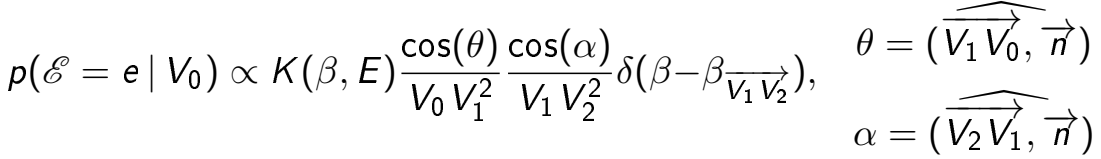

$$
\begin{aligned}
& \beta_{\overrightarrow{V_{1} V_{2}}}:=\left(\overrightarrow{V_{1} \vec{V}_{2}, \overrightarrow{O V_{1}}}\right)
\end{aligned}
$$




\section{Simulation setup}

Camera : three Si layers, $32 \times 32 \times 0.5 \mathrm{~cm}^{3}$, altitude $z \in\{-5,-6,-7\}$, absorber made of $0.5 \times 0.5 \times 4 \mathrm{~cm}^{3}$ crystals at $z=-17$

Source : mono-energetic (364 keV) point source in $\mathrm{O}(0,0,0), 10^{8}$ emitted photons.

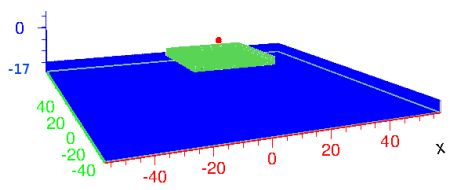
Events : $\approx 7 \times 10^{6}$

Ideal detectors :

- $E_{1}+E_{2}=E \rightarrow e:=\left(V_{1}, V_{2}, \beta\right)$

- The sequence of interactions is known

- The $(x, y)$ positions of interaction are measured without noise; the $z$ coordinate is taken at half-depth of the detector.

The number of events/bin $\left(\approx 1 \mathrm{~mm}, \approx 1^{\circ}\right)$ is statistically small. 


\section{Averaging}

(1) Selection of events having $z_{V_{1}}=-5 \mathrm{~cm}$ and $z_{V_{2}}=-6 \mathrm{~cm}$.

(2) For each $r_{1} \in(0,16 \mathrm{~cm})$, selection of $V_{1} \in C\left(O_{1}, r_{1}\right)$ parametrized by $\varphi_{1} \in[0,2 \pi)$.

(3) For each $V_{1}$ and for each $r_{2} \in(0,8 \mathrm{~cm})$, selection of $V_{2} \in C\left(W_{2}, r_{2}\right)$ parametrized by $\varphi_{2} \in[0,2 \pi)$.

(4) For each $V_{1}, V_{2}$ there is one single $\beta$ such that $V_{0} \in \mathscr{C}\left(V_{1}, V_{2}, \beta\right)$ When

$$
\begin{gathered}
p\left(V_{1}, V_{2}, \beta \mid V_{0}\right) \propto K(\beta, E) \frac{\cos (\theta)}{V_{0} V_{1}^{2}} \frac{\cos (\alpha)}{V_{1} V_{2}^{2}} \delta\left(\beta-\beta \overrightarrow{V_{1} V_{2}}\right) \\
\sum_{V_{1} \in C\left(O_{1}, r_{1}\right)} \sum_{V_{2} \in C\left(W_{2}, r_{2}\right)} \sum_{\beta} \frac{\widehat{p}\left(V_{1}, V_{2}, \beta \mid V_{0}\right)}{K(\beta, E)}
\end{gathered}
$$

should be proportional to

$$
M\left(r_{1}, r_{2}\right)=r_{1} r_{2} \frac{\cos \left(\theta_{r_{2}}\right)}{d_{1}^{2}} \frac{\cos \left(\alpha_{r_{1}}\right)}{d_{2}^{2}} .
$$

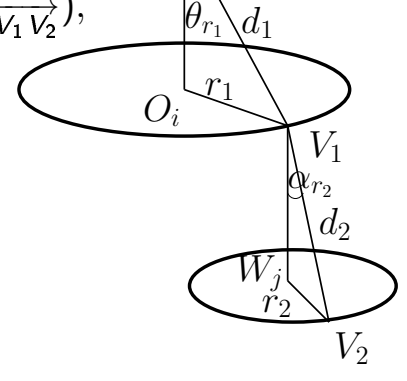




\section{Numerical investigation}

Camera : three Si layers $z \in\{-5,-6,-7\}$, absorber at $z=-17$

Source : mono-energetic (364 keV) point source in $\mathrm{O}(0,0,0), 10^{8}$ emitted particles Events : $\approx 7 \times 10^{6}$

Binning : $d_{r_{1}}=d_{r_{2}}=2 \mathrm{~mm}, d_{\beta}=3^{\circ}$

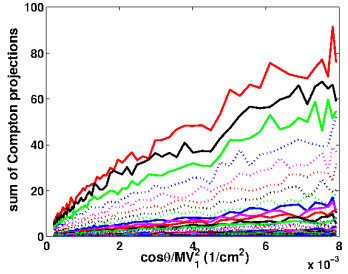

Variation of $\frac{M\left(r_{1}, r_{2}\right)}{r_{1} r_{2}}$
w.r. to $\frac{\cos \theta_{r_{1}}}{d_{1}^{2}} ;$

Each $r_{2}$ : a curve

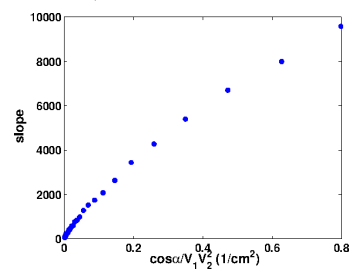

Slope from regression as function of $\frac{\cos \alpha_{r_{2}}}{d_{2}^{2}}$;

Each $r_{2}$ : a point
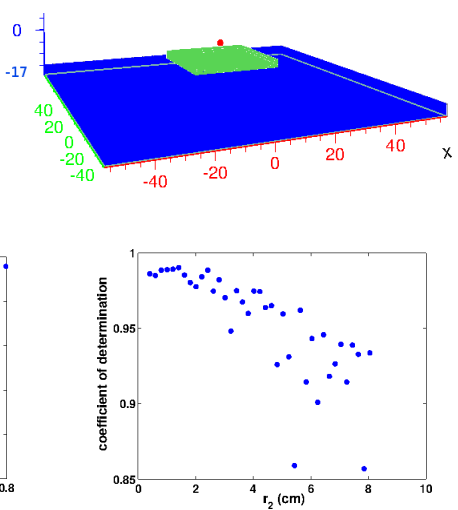

Quality of the regression. Degrades as $V_{2}$ moves tw. the border. 


\section{Summary}

(1) SPECT imaging with the Compton camera

(2) From the events to the image: the system matrix

(3) Image reconstruction : list-mode MLEM algorithm 
Image reconstruction : LM-MLEM algorithm

$$
\widehat{\lambda}_{j}^{(\ell+1)}=\frac{\widehat{\lambda}_{j}^{(\ell)}}{s_{j}} \sum_{i} \frac{t_{i j}}{\sum_{k} t_{i k} \widehat{\lambda}_{k}^{(\ell)}}
$$

where $t_{i j}$ is the probability for a photon emitted by the voxel $j$ to be detected as event $e_{i}$,

$$
t_{i j}=p\left(\mathscr{E}=e_{i} \mid v_{j}\right)
$$

and $s_{j}$ is the probability for a photon emitted by the voxel $j$ to be detected. Thus,

$$
s_{j}=\sum_{i} t_{i j}
$$

where the sum is taken on all possible events, not only on the realized ones. We take

$$
t_{i j}=\frac{\cos \left(\alpha_{i}\right)}{V_{1} V_{2}^{2}} \int_{M \in v_{j}} K\left(\beta_{M}, E\right) \frac{\cos \left(\theta_{M}\right)}{V_{1} M^{2}} g\left(\beta_{M} \mid \beta_{i}, \sigma_{\beta_{i}}\right) d v .
$$




\section{Sensitivity matrix}
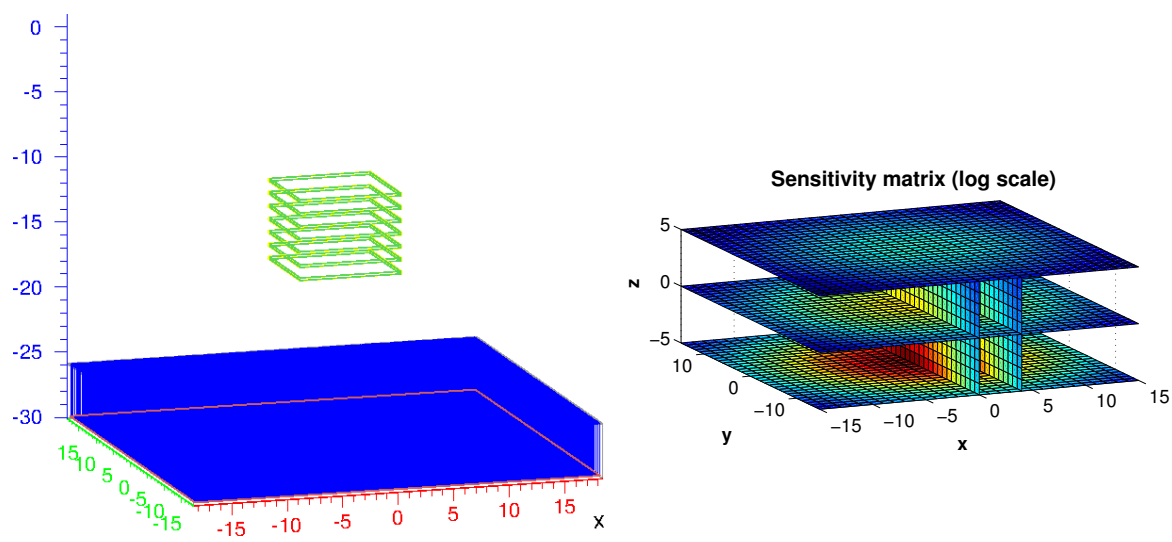

- Si scatterers, $9 \times 9 \times 0.2 \mathrm{~cm}^{3}, 128 \times 128$ strips, energy resolution 2.35 keV FWHM

- absorber in LYSO crystals, $0.5 \times 0.5 \times 4 \mathrm{~cm}^{3}$, energy resolution function of the energy of the incident particle, $31 \mathrm{keV} @ 1 \mathrm{MeV}$. 


\section{Summary}

(1) SPECT imaging with the Compton camera

(2) From the events to the image: the system matrix

(3) Image reconstruction: list-mode MLEM algorithm

(4) Numerical results 


\section{Aim}

(1) Role of the parameters of the system matrix

(2) Role of the sensitivity matrix

(3) Joint influence of the system matrix and of the sensitivity 
Joint influence of the model and sensitivity

Mono-energetic $(1275 \mathrm{keV})$ simulated point source in $(10,0,0)$.

No energy selection, 3500 events, 20 iterations.

- System matrix from the model :

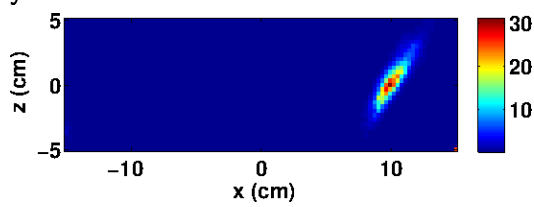

$s_{j}$ from MC simulation

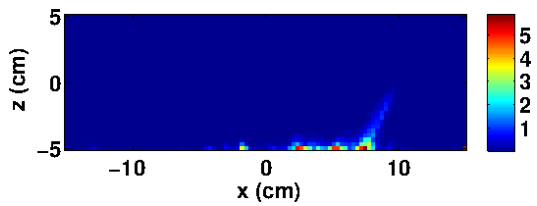

$s_{j} \equiv 1$

- Elements of the system matrix set to one :

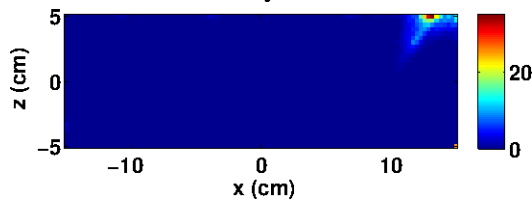

$s_{j}$ from MC simulation

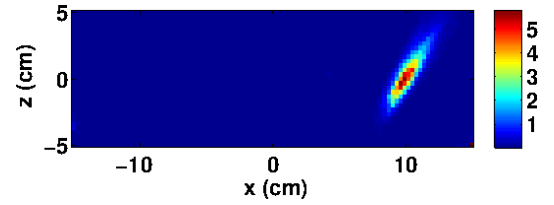

$s_{j} \equiv 1$ 
Is the model of the system matrix gainful?

Mono-energetic $(1275 \mathrm{keV})$ line source $(y \in[-14,5])$ at $10 \mathrm{~cm}$ from the camera. Energy selection (20\%), 6000 events, 20 iterations.
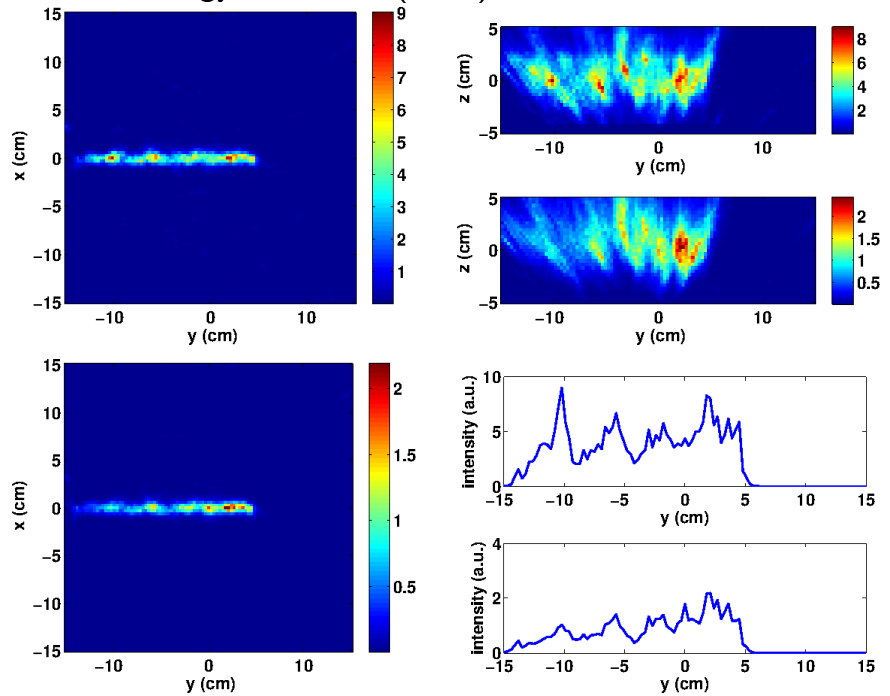

The 3D image of the source was calculated on the base of the proposed model (upper image from each pair), then with $t_{i j} \equiv 1$ and $s_{j} \equiv 1$ (lower image from each pair). 


\section{Examples}

Mono-energetic (1275 keV) Shepp-Logan phantom at $10 \mathrm{~cm}$ from the camera. Energy selection (20\%), 80000 events, 20 iterations.

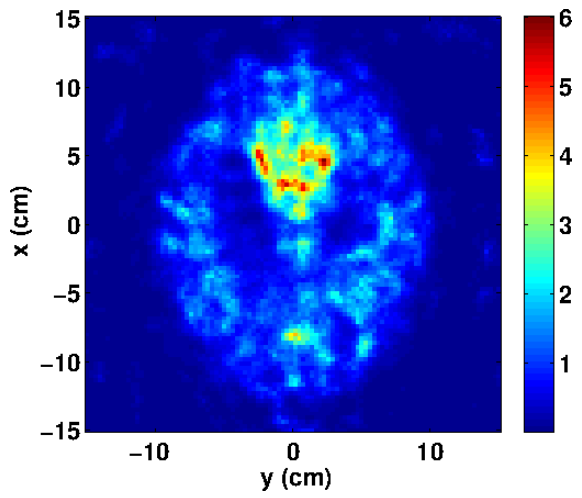

Reconstructed image.

Horizontal slice

at $10 \mathrm{~cm}$ from the camera.

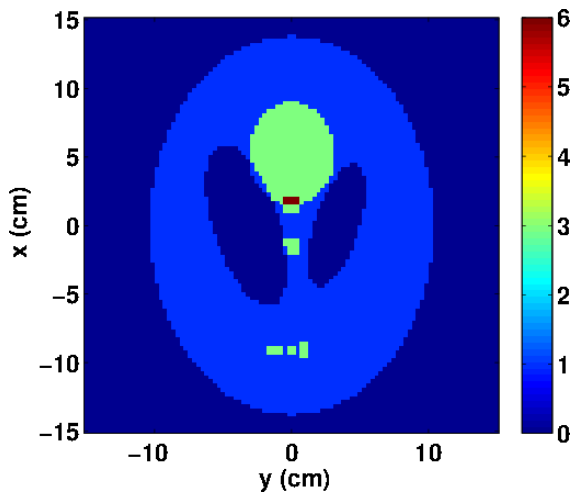

Original phantom (2D) 


\section{Conclusions}

- The quality of the Compton images are strongly related to the models chosen for the system matrix and to the sensitivity matrix.

- The proposed theoretical model of the system matrix for ideal detectors is confirmed by simulations.

- Variants of the iterative reconstruction algorithm may improve the quality of the images.

This work was supported in part by the ENVISION Project co-funded by the European Commission under the FP7 Collaborative Projects, in part by the ETOILE Research Program $P R R H / U C B L$, and in part by the LABEX PRIMES of the Universite de Lyon. 